\title{
Making space for harmonic oscillators
}

\author{
Leo Michelotti
}

July 30, 2004

\begin{abstract}
If we restrict the number of harmonic oscillator energy eigenstates to some finite value, $N$, then the discrete spectrum of the corresponding position operator comprise the roots of the Hermite polynomial $H_{N+1}$. Its range is just large enough to accomodate classical motion at high energy. A negative energy term must be added to the Hamiltonian which affects only the last eigenstate, $|N\rangle$, suggesting it is concentrated at the extrema of this finite "space." Calculations support a conjecture that, in the limit of large $N$, the global distribution of points approaches the differential form for classical action.
\end{abstract}


The notion of a vacuum is not only in which is nothing, but also implies a space capable of holding a body and in which there is not a body.... Whereas we hold that there was no place or space before the world was.

- St. Thomas Aquinas

Summa Theologica

Question 46: Reply Obj.4

These were remarkable deductions: (i) empty space is not nothing but something, and (ii) the universe and its space came into being simultaneously. Without presuming to investigate how One might have accomplished that, we shall briefly consider the harmonic oscillator. Its quantum theory comes equipped with conveniently named "annihilation" and "creation" operators which, if used as the primary objects, provide a way of describing physics without presupposing a physical "space." In this view, "space" is a derived entity.

The content is divided as follows. Fundamentals of the harmonic oscillator are tersely written in the first section for purposes of notation and reference. In the next, we introduce approximations for the operators as a preliminary to doing calculations, and in the third and fourth we examine the spectra of the corresponding position operators, a "space" constructed specifically for a universe of harmonic oscillators. The final section contains a brief synopsis.

The work reported herein was undertaken as a diversion; it possesses nothing erudite or scholarly. In fact, it is little more than a variation on Problem XII.3 of Messiah's textbook Quantum Mechanics [2], which asks students to “[s]et up the eigenvalue problem of $q$ in [the number representation]; show that the spectrum of $q$ is nondegenerate continuous and extends from $-\infty$ to $\infty$." Nonetheless, the results may be interesting in themselves. If nothing else, in the process of going through all of this we shall develop a conjecture on the roots of Hermite polynomials that might possibly be new.

\section{Preliminaries: the Harmonic Oscillator}

For later reference, we collect here a few facts about the harmonic oscillator, split according to the classical and quantum theories. Everyone learns these facts within the standard physics curriculum, so we shall not pause for explanation or development. ${ }^{1}$ This section may be omitted and referred to as needed.

\subsection{Classical Mechanics}

The linear differential equations describing a harmonic oscillator can be written in matrix form.

$$
\frac{d}{d t}\left(\begin{array}{c}
x \\
p
\end{array}\right)=\left(\begin{array}{cc}
0 & 1 / m \\
-m \omega^{2} & 0
\end{array}\right) \cdot\left(\begin{array}{l}
x \\
p
\end{array}\right)
$$

Variables $x$ and $p$ are the dynamical coordinates, position and momentum respectively; $m$ is the mass of the oscillator, $\omega$ is its (radial) frequency, and $t$ represents time, whatever that means. This is easily diagonalized,

$$
\left(\begin{array}{cc}
0 & 1 / m \\
-m \omega^{2} & 0
\end{array}\right) \cdot\left(\begin{array}{cc}
-1 / \sqrt{2 m \omega} & -1 / \sqrt{2 m \omega} \\
i \sqrt{m \omega / 2} & -i \sqrt{m \omega / 2}
\end{array}\right)=\left(\begin{array}{cc}
-1 / \sqrt{2 m \omega} & -1 / \sqrt{2 m \omega} \\
i \sqrt{m \omega / 2} & -i \sqrt{m \omega / 2}
\end{array}\right) \cdot\left(\begin{array}{cc}
-i \omega & 0 \\
0 & i \omega
\end{array}\right)
$$

so that, if we define complex coordinates, $a$ and $a^{*}$ as follows,

$$
\left(\begin{array}{c}
x \\
p
\end{array}\right)=\left(\begin{array}{cc}
-1 / \sqrt{2 m \omega} & -1 / \sqrt{2 m \omega} \\
i \sqrt{m \omega / 2} & -i \sqrt{m \omega / 2}
\end{array}\right) \cdot\left(\begin{array}{c}
a \\
a^{*}
\end{array}\right),
$$

\footnotetext{
${ }^{1}$ Some of the text has been copied directly from Ref. [3].
} 
or inverted,

$$
\left(\begin{array}{c}
a \\
a^{*}
\end{array}\right)=\left(\begin{array}{cc}
\sqrt{m \omega / 2} & i / \sqrt{2 m \omega} \\
\sqrt{m \omega / 2} & -i / \sqrt{2 m \omega}
\end{array}\right) \cdot\left(\begin{array}{c}
x \\
p
\end{array}\right),
$$

then the equation of motion takes the diagonal form,

$$
\frac{d a}{d t}=-i \omega a
$$

The pair $\left(a, a^{*}\right)^{T}$ are the "Weyl coordinates" of the system. ${ }^{2}$

In the Hamiltonian formalism, the harmonic oscillator is described by a quadratic form,

$$
\begin{aligned}
H & =\frac{p^{2}}{2 m}+\frac{1}{2} m \omega^{2} x^{2} \\
& =\omega(\sqrt{m \omega / 2} x-i p / \sqrt{2 m \omega})(\sqrt{m \omega / 2} x+i p / \sqrt{2 m \omega}) \\
& =\omega a^{*} a .
\end{aligned}
$$

Further, we can express Weyl coordinates in terms of angle-action coordinates, ${ }^{3}(\varphi, I)$, as follows.

$$
a=i \sqrt{I} e^{-i \varphi}, \quad a^{*}=-i \sqrt{I} e^{i \varphi}
$$

On this coordinate chart, the Hamiltonian takes on the form,

$$
H=\omega I \text {. }
$$

\subsection{Quantum Mechanics}

In quantum mechanics, the harmonic oscillator's Hamiltonian possesses the same quadratic form as Eq.(1), with all variables now evaluated as Hermitian operators on a Hilbert space rather than real numbers.

$$
\mathbf{H}=\frac{\mathbf{p}^{2}}{2 m}+\frac{1}{2} m \omega^{2} \mathbf{x}^{2}
$$

Dimensionless variables are introduced in order to "avoid cluttering the calculations by useless constants."

$$
\begin{aligned}
\mathbf{u} & \equiv \sqrt{\frac{m \omega}{\hbar}} \mathbf{x}=\left(\mathbf{a}+\mathbf{a}^{\dagger}\right) / \sqrt{2} \\
\mathbf{v} & \equiv \sqrt{\frac{1}{m \hbar \omega}}(i \mathbf{p})=\left(\mathbf{a}-\mathbf{a}^{\dagger}\right) / \sqrt{2}
\end{aligned}
$$

Their quantum commutators are related as follows.

$$
[\mathbf{x}, \mathbf{p}]=i \hbar \quad \Rightarrow \quad[\mathbf{u}, \mathbf{v}]=-\mathbf{1}
$$

\footnotetext{
${ }^{2}$ I cannot remember from what textbook I picked up this piece of information.

${ }^{3}$ We mention in passing that, unlike $(x, p)$, angle-action coordinates are defined over a punctured phase space from which the origin has been removed, making it topologically equivalent to a cylinder, not the Euclidean plane.

${ }^{4}$ From Chapter XII, Section 2. of Ref.[2].
} 


$$
\left[\mathbf{a}, \mathbf{a}^{\dagger}\right]=\frac{1}{2}[\mathbf{u}+\mathbf{v}, \mathbf{u}-\mathbf{v}]=[\mathbf{v}, \mathbf{u}]=\mathbf{1}
$$

Finally, we rewrite the Hamiltonian using factorization.

$$
\begin{aligned}
\mathbf{H} & =\frac{1}{2} \hbar \omega\left(\mathbf{u}^{2}+\mathbf{v}^{2}\right) \\
& =\frac{1}{2} \hbar \omega((\mathbf{u}-\mathbf{v})(\mathbf{u}+\mathbf{v})-[\mathbf{u}, \mathbf{v}]) \\
& =\hbar \omega\left(\mathbf{a}^{\dagger} \mathbf{a}+1 / 2\right)
\end{aligned}
$$

The number representation is the set of eigenstates of $\mathbf{H}$, equivalently of $\mathbf{a}^{\dagger} \mathbf{a}$, which comprise an orthonormal basis for a Hilbert space.

$$
\mathbf{a}^{\dagger} \mathbf{a}|n\rangle=n|n\rangle, \quad n=0,1,2,3, \ldots
$$

Any other set of eigenstates is frowned upon, as it would contain an infinite set of states with unbounded negative energy. Their representation, in dimensionless coordinates, involves the Hermite polynomials, $H_{n}$.

$$
\begin{aligned}
\langle u \mid n\rangle=\psi_{n}(u) & =\left(\sqrt{\pi} 2^{n} n !\right)^{-1 / 2} e^{-u^{2} / 2} H_{n}(u) \\
\int_{-\infty}^{\infty} d u \psi_{n}(u) \psi_{m}(u) & =\delta_{m n} \\
\sum_{n=0}^{\infty} \psi_{n}\left(u_{1}\right) \psi_{n}\left(u_{2}\right) & =\delta\left(u_{1}-u_{2}\right)
\end{aligned}
$$

\section{Truncated Operators}

Quantum physics of a harmonic oscillator is encoded into a sequence of square roots of integers, as expressed by the number representation of the annihilation operator: $\langle m|\mathbf{a}| n\rangle=\delta_{m+1, n} \sqrt{n}$, or written as a semi-infinite matrix,

$$
\mathbf{a}=\left(\begin{array}{cccccc}
0 & \sqrt{1} & 0 & 0 & 0 & \ldots \\
0 & 0 & \sqrt{2} & 0 & 0 & \ldots \\
0 & 0 & 0 & \sqrt{3} & 0 & \ldots \\
0 & 0 & 0 & 0 & \sqrt{4} & \ldots \\
0 & 0 & 0 & 0 & 0 & \ldots \\
\vdots & \vdots & \vdots & \vdots & \vdots & \ddots
\end{array}\right)
$$

We shall truncate this and study properties of its $(N+1) \times(N+1)$ submatrix,

$$
\mathbf{a}=\left(\begin{array}{ccccccc}
0 & \sqrt{1} & 0 & 0 & 0 & \ldots & 0 \\
0 & 0 & \sqrt{2} & 0 & 0 & \ldots & 0 \\
0 & 0 & 0 & \sqrt{3} & 0 & \ldots & 0 \\
0 & 0 & 0 & 0 & \sqrt{4} & \ldots & 0 \\
0 & 0 & 0 & 0 & 0 & \ldots & 0 \\
\vdots & \vdots & & \vdots & \vdots & \vdots & \vdots \\
0 & 0 & \ldots & 0 & 0 & 0 & \sqrt{N} \\
0 & 0 & \ldots & 0 & 0 & 0 & 0
\end{array}\right) .
$$


The notations $\mathbf{a}, \mathbf{u}, \mathbf{H}$, and so forth, will be retained with the understanding that, from this point on, they represent finite matrices. If justification for this truncation is needed, one might argue that it is a preliminary to writing computer programs: if lattice gauge theorists can profitably do calculations on a space of 512 nodes, why may we not consider one consisting of 512 energy levels? Another argument can be presented on the basis of prudential parsimony. Suppose that a universe consisting of harmonic oscillators contains only a finite amount of energy. Why should it then possess an infinite number of energy levels? What is the point of all those states which cannot possibly be occupied?

After truncation, $\mathbf{a}^{\dagger} \mathbf{a}$ is still diagonal, with the same eigenvalues as before, so we retain the subset $\{|0\rangle,|1\rangle,|2\rangle, \ldots|N\rangle\}$ of the number representation as a basis set of states. However, commutations relations must be modified. Because $\operatorname{Tr}(\mathbf{A B})=\operatorname{Tr}(\mathbf{B A})$ the commutator of any two finite matrices must vanish. Thus, instead of the usual commutation relations of Eq.(4), the truncated $(N+1) \times(N+1)$ matrices $\mathbf{a}$ and $\mathbf{a}^{\dagger}$ satisfy,

$$
\left[\mathbf{a}, \mathbf{a}^{\dagger}\right]=\mathbf{1}-(N+1)|N\rangle\langle N|
$$

The commutators of $\mathbf{u}$ and $\mathbf{v}$ must also be modified.

$$
[\mathbf{u}, \mathbf{v}]=\frac{1}{2}\left[\mathbf{a}+\mathbf{a}^{\dagger}, \mathbf{a}-\mathbf{a}^{\dagger}\right]=\left[\mathbf{a}^{\dagger}, \mathbf{a}\right]=-\mathbf{1}+(N+1)|N\rangle\langle N|
$$

Its effect on the Hamiltonian is as follows.

$$
\begin{aligned}
\mathbf{H} & =\frac{1}{2} \hbar \omega\left(\mathbf{u}^{2}+\mathbf{v}^{2}\right) \\
& =\frac{1}{2} \hbar \omega((\mathbf{u}-\mathbf{v})(\mathbf{u}+\mathbf{v})-[\mathbf{u}, \mathbf{v}]) \\
& =\hbar \omega\left(\mathbf{a}^{\dagger} \mathbf{a}+\frac{1}{2}(\mathbf{1}-(N+1)|N\rangle\langle N|)\right)
\end{aligned}
$$

All but one of the matrix elements of $\mathbf{H}$ are unaffected by truncation. The only one that must be adjusted is the final diagonal element.

$$
\langle N|\mathbf{H}| N\rangle=\frac{1}{2} N \hbar \omega
$$

Because of truncation, $|N-1\rangle$, not $|N\rangle$, is the highest energy state. The latter is reduced in energy by $\frac{1}{2}(N+1) \hbar \omega$ compared to the value it would have had in the infinite context. Effectively, for large but finite $N$, an enormous pool of negative energy has been introduced. Because only $|N\rangle$ is sensitive to this pool, it must be concentrated where $\langle u \mid N\rangle$ is large, i.e. at large values of $u$.

As a minor exercise of no particular significance, suppose that one oscillator is placed in each energy level. The total energy would be,

$$
\begin{aligned}
E_{\text {tot }} / \hbar \omega & =\sum_{n=0}^{N}\left\langle n\left|\left(\mathbf{a}^{\dagger} \mathbf{a}+\frac{1}{2}(\mathbf{1}-(N+1)|N\rangle\langle N|)\right)\right| n\right\rangle \\
& =\sum_{n=0}^{N} n+\frac{1}{2}\left(\left(\sum_{n=0}^{N} 1\right)-(N+1)\right) \\
& =\frac{1}{2} N(N+1)
\end{aligned}
$$

The extra negative energy term cancels the additional zero-point energy offset from all the states. 


\section{The Spectrum of Space}

"Space" is that which is measured by the position operator. Its spectrum consists of the eigenvalues of $\mathbf{x}$, equivalently $\mathbf{u}$ as defined in Eq.(3). In the context of a truncated $\mathbf{u}$, we shall call its spectrum " $N$-space."

The eigenvector of $\mathbf{u}$ corresponding to eigenvalue $\lambda$ satisfies the equation,

$$
\left(\begin{array}{cccccc}
0 & \sqrt{1} & 0 & 0 & \cdots & 0 \\
\sqrt{1} & 0 & \sqrt{2} & 0 & \cdots & 0 \\
0 & \sqrt{2} & 0 & \sqrt{3} & \cdots & 0 \\
0 & 0 & \sqrt{3} & 0 & \cdots & 0 \\
\vdots & \vdots & & & & \vdots \\
0 & 0 & \cdots & \sqrt{N-1} & 0 & \sqrt{N} \\
0 & 0 & \cdots & 0 & \sqrt{N} & 0
\end{array}\right) \cdot\left(\begin{array}{l}
u_{0} \\
u_{1} \\
u_{2} \\
u_{3} \\
\vdots \\
u_{N-1} \\
u_{N}
\end{array}\right)=(\sqrt{2} \lambda)\left(\begin{array}{l}
u_{0} \\
u_{1} \\
u_{2} \\
u_{3} \\
\vdots \\
u_{N-1} \\
u_{N}
\end{array}\right)
$$

A similar equation can be written for $\mathbf{v}$ by negating each element on the lower diagonal. Without actually solving anything, a few conclusions can be drawn from the structure of this equation alone.

1. If $\lambda$ is an eigenvalue of $\mathbf{u}$, then so is $-\lambda$

2. If $\lambda$ is an eigenvalue of $\mathbf{u}$, then $i \lambda$ is an eigenvalue of $\mathbf{v}$.

3. Zero is an eigenvalue if and only if $N$ is even.

These three properties follow as consequences of lemmas proven in an appendix. The second one means that - modulo the difference in scale factors $-\mathbf{x}$ and $\mathbf{p}$ possess the same eigenvalues. Because of the third, I am biased toward even values of $N$ : their phase spaces come pre-punctured in anticipation of angle-action variables.

Written one row at a time, the eigenvalue eqution, Eq.(8), becomes a recurrence relation,

$$
\sqrt{n+1} u_{n+1}=(\sqrt{2} \lambda) u_{n}-\sqrt{n} u_{n-1}, \quad n=0 \ldots N,
$$

with the convention that $u_{-1}=u_{N+1}=0$. It can be put into more familiar form by multiplying both sides by $\sqrt{2^{n+1} n !}$.

$$
\left(\sqrt{2^{n+1}(n+1) !} u_{n+1}\right)=(2 \lambda)\left(\sqrt{2^{n} n !} u_{n}\right)-(2 n)\left(\sqrt{2^{n-1}(n-1) !} u_{n-1}\right) .
$$

To no one's surprise, this is a recurrence satisfied by Hermite polynomials. Normalization is arbitrary, of course. If we fix it by setting $u_{0} \equiv 1$, then the recursion for identifies the components $u_{1}, u_{2}, \ldots u_{N-1}$ as,

$$
u_{n}=\sqrt{\frac{1}{2^{n} n !}} H_{n}(\lambda)
$$

Instead, if we set $u_{0} \equiv \pi^{-1 / 4} e^{-\lambda^{2} / 2}$, then

$$
u_{n}=\psi_{n}(\lambda)
$$

the quantum wave function with normalization as written in Eq.(5).

However, our interest here lies more in the eigenvalues than their eigenvectors. The final step of the recursion Eq.(9) is,

$$
0=(2 \lambda)\left(\sqrt{2^{N} N !} u_{N}\right)-(2 N)\left(\sqrt{2^{N-1}(N-1) !} u_{N-1}\right) .
$$


Because $u_{N}$ and $u_{N-1}$ are determined by previous conditions, this serves as a self-consistency equation for the eigenvalue, $\lambda$. At the same time, we have established that $\sqrt{2^{n} n !} u_{n} \propto H_{n}(\lambda)$, for $n=0,1, \ldots, N$. Thus, the right-hand side of Eq.(10) implies that

$$
H_{N+1}(\lambda)=0 .
$$

Based on known properties of Hermite polynomials, we could immediately infer the three properties already written on the previous page. To them we add:

(4) The characteristic polynomial of $\mathbf{u}$ is

$$
\operatorname{det}[\mathbf{u}-\lambda \mathbf{1}]=(-1 / 2)^{N+1} \cdot H_{N+1}(\lambda) .
$$

Equating leading order terms on both sides determines the proportionality constant.

Positive eigenvalues of $\mathbf{u}$, obtained by numerical diagonalization, are plotted in Figure 1 for $N=1 \ldots 64$. The spectra for even values of $N$ are drawn in black; for odd, in red. The lines, meant to guide the eye, follow "trajectories" of eigenvalues

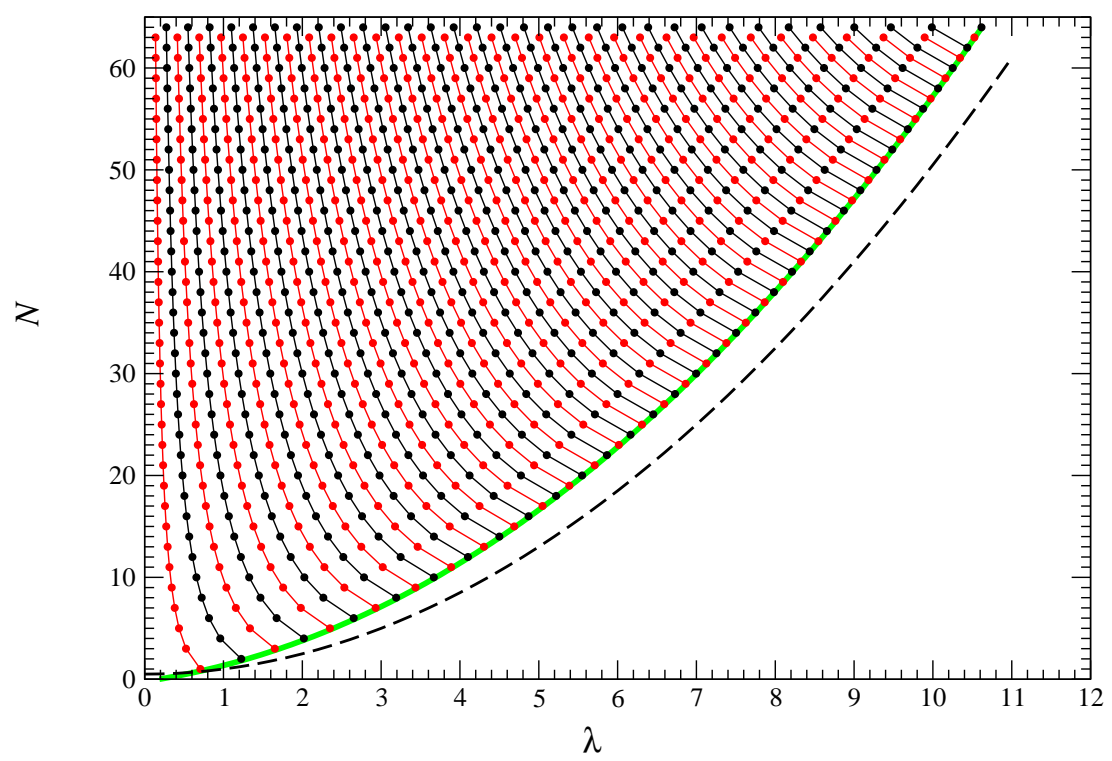

Figure 1: Positive eigenvalues of $\mathbf{u}$ for $N=1 \ldots 64$. The solid green and dashed black curves indicate two estimates for the maximum eigenvalue.

from their points of creation through $N=64$. Two observations deserve closer scrutiny: (a) the maximum eigenvalue grows slowly with $N$ and (b) eigenvalues grow closer together as $N$ increases.

(a) A semi-classical argument suggests that $\lambda_{\max }$ can be approximated,

$$
\lambda_{\max } \approx \sqrt{2 N-1}
$$

This follows by noting that the maximum reach of a classical harmonic oscillator is given by $E=m \omega^{2} x_{\max }^{2} / 2$. Because of Eq.(7), the largest energy eigenstate is $|N-1\rangle$. Setting $E=(N-1 / 2) \hbar \omega$, then produces the result, which appears as the dashed curve in Figure 1. 
A more exact estimate is obtained by using asymptotic expressions for the maximum roots of Hermite polynomials. According to Szegö [4], Equation (6.32.5), the largest root of $H_{n}(\lambda)$ satisfies,

$$
\lambda_{\max }=\sqrt{2 n+1}-\frac{i_{1}+\varepsilon_{n}}{(6 \sqrt{2 n+1})^{1 / 3}}
$$

where $i_{1}$ is the smallest (in fact, positive) real root of Airy's function and $\lim _{n \rightarrow \infty} \varepsilon_{n}=0 .{ }^{5}$ We replace $n$ with $N+1$ (see Eq.(11)), corrupt this beautiful expression a bit by ignoring the error term, $\varepsilon_{n}$, and state that the maximum reach of $N$-space, for large $N$, must be approximately

$$
\lambda_{\max } \approx \sqrt{2 N+3}-\frac{1.85575}{(\sqrt{2 N+3})^{1 / 3}}
$$

This appears as the solid green curve in Figure 1. Its close agreement with the numerically calculated values is remarkable, considering it arises from an approximation to an expression valid for large $N$.

On a personal note, despite the obvious superiority of the asymptotic formula, the argument from classical mechanics is more appealing. There is something pleasingly economical (or "surprisingly parsimonious" or "impressively prudent") about $N$-space being just about the right size to accomodate classical dynamics at high energy. It has "enough space" but no "wasted space." There is no a priori reason why this should be so, but it is.

(b) Intuitively we expect the spatial eigenvalues to cluster indefinitely closer together as $N$ increases. This is correct, although it is difficult to tell from Figure 1 whether they instead achieve a limiting separation. To settle the issue, we again appeal to an asymptotic formula. ${ }^{6}$

$$
\text { For any fixed } x: \lim _{n \rightarrow \infty} \frac{(-1)^{n}}{4^{n} n !}\left\{\begin{array}{c}
\sqrt{n} H_{2 n}(x /(2 \sqrt{n})) \\
H_{2 n+1}(x /(2 \sqrt{n}))
\end{array}\right\}=\frac{1}{\sqrt{\pi}}\left\{\begin{array}{c}
\cos (x) \\
2 \sin (x)
\end{array}\right\} .
$$

Thus, within a finite interval containing the origin, the spacing between successive eigenvalues at truncation step $N$ approaches the limit,

$$
\delta \lambda=\frac{\pi}{\sqrt{2}}\left\{\begin{array}{cl}
\frac{1}{\sqrt{N+1}}, & N \text { odd } \\
\frac{1}{\sqrt{N}}, & N \text { even }
\end{array}\right.
$$

Thus, $\delta \lambda \rightarrow 0$ as $N \rightarrow \infty$, albeit slowly. This means that, by choosing $N$ sufficiently large, we can place an arbitrarily large number of eigenvalues as close as desired to any given real number. In this sense, the set of eigenvalues do approach the real axis continuum. Moreover, the density of eigenvalues becomes uniform on any local neighborhood of the origin: that is, $\delta \lambda$ becomes independent of $\lambda$, as is seen in Eq.(13). However, as we shall see in the next section, their global density does not.

\section{Spatial Spectra Distribution Functions}

Cumulative distributions of the spatial spectra are plotted in Figure 2. The plot on the left shows

$$
F(\lambda)=\text { number of eigenvalues of } \mathbf{u} \leq \lambda .
$$

for $N=2^{m}, m=4 \ldots 8$. On the right are displayed the same data normalized according to the maximum eigenvalue and the

\footnotetext{
${ }^{5}$ Szegö noted that the numerical value $6^{-1 / 3} i_{1}=1.85575 \ldots$ "cannot be replaced by a smaller [constant]" in this expression. He also wrote that this result "possess[es] an extended literature" and provided several references to preceding works.

${ }^{6}$ Taken from Equations (22.15.3) and (22.15.4) of Reference [1].
} 

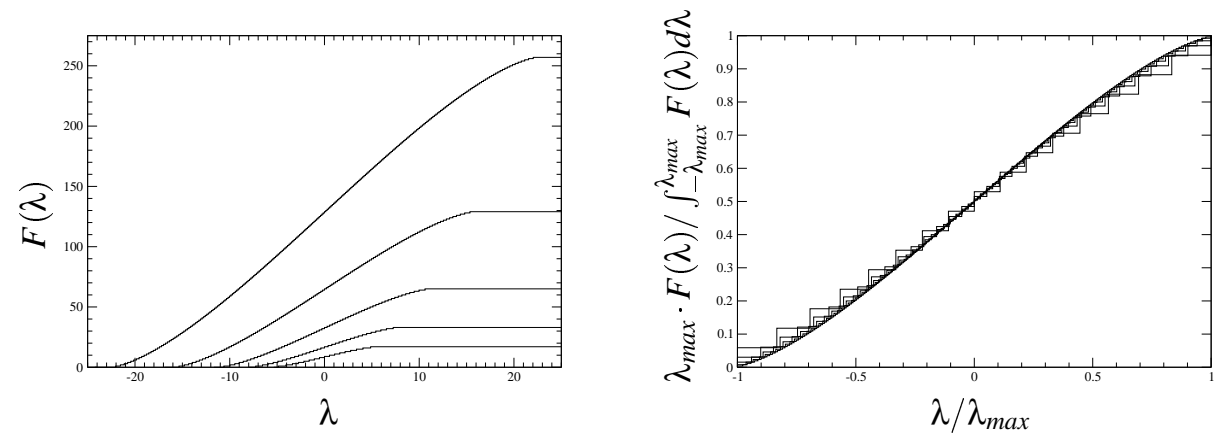

Figure 2: Cumulative distributions of the eigenvalues of $\mathbf{u}$ : (a) unnormalized and (b) normalized.

area under the curve. That is, the abscissa marks $\lambda / \lambda_{\max }$ while the ordinate is

$$
\frac{\lambda_{\max } \cdot F(\lambda)}{\int_{-\lambda_{\max }}^{\lambda_{\max }} F(\lambda) d \lambda} .
$$

This normalizes the area under the curves to unity.

Somewhat more revealing is the normalized differential distribution,

$$
\frac{\lambda_{\max }}{F\left(\lambda_{\max }\right)} \cdot \frac{d F}{d \lambda}=\frac{1}{N+1} \cdot \frac{d F}{d\left(\lambda / \lambda_{\max }\right)}
$$

where we recognize that $F\left(\lambda_{\max }\right)=N+1$. Our numerical approximation for $N$-space is $d F / d \lambda \approx \Delta F / \Delta \lambda=1 / \Delta \lambda$. This is shown in Figure 3; the black curves are interpolations of the sets of ordered pairs,

$$
\left(\frac{1}{\lambda_{\max }} \frac{\lambda_{i}+\lambda_{i-1}}{2}, \frac{\lambda_{\max }}{N+1} \frac{1}{\lambda_{i}-\lambda_{i-1}}\right) \text {, for } i=1,2, \ldots, N, \text { and } N=2^{m}, m=4,5, \ldots 9 \text {. }
$$

As $N$ increases, these distributions appear to approach a limit, displayed in Figure 3 as the green curve. Since I have no proof, I'll call this a

$$
\text { CONJECTURE : } \lim _{N \rightarrow \infty} \frac{\lambda_{\max }}{F\left(\lambda_{\max }\right)} \cdot \frac{d F(\lambda)}{d \lambda}=\frac{2}{\pi} \cdot \sqrt{1-\left(\lambda / \lambda_{\max }\right)^{2}}
$$

The constant $2 / \pi$ is determined by the fact that the integral of the normalized distribution must be one.

Assuming that this conjecture is correct, we can develop a (semi-classical) physical interpretation for it. Recall that $\lambda$ is an eigenvalue of $\mathbf{u}$ and consider the following.

$$
\begin{aligned}
\sqrt{\lambda_{\max }^{2}-\lambda^{2}} & =\sqrt{\frac{m \omega}{\hbar} x_{\max }^{2}-\frac{m \omega}{\hbar} x^{2}} \\
& =\sqrt{\frac{2}{\hbar \omega}} \sqrt{\frac{1}{2} m \omega^{2} x_{\max }^{2}-\frac{1}{2} m \omega^{2} x^{2}} \\
& =\sqrt{\frac{2}{\hbar \omega}} \sqrt{\frac{p^{2}}{2 m}} \\
& =\frac{1}{\sqrt{m \hbar \omega}}|p|
\end{aligned}
$$




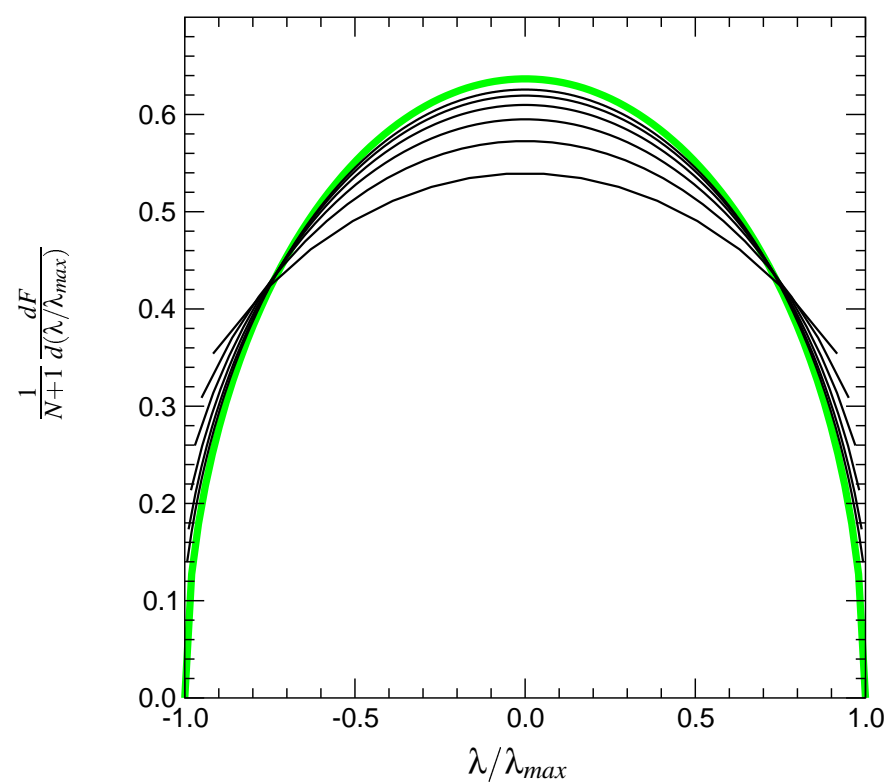

Figure 3: Differential distribution of points in the $N$-space, for $N=16,32,64,128,256$, and 512. The green curve, whose form is given in the text, is conjectured to be the limiting distribution.

Here, $|p|$ is evaluated on the orbit for which $E=\frac{1}{2} m \omega^{2} x_{\max }^{2}$. Combining this with the conjecture, we can interpret $d F$ as follows.

$$
\begin{aligned}
d F & =\frac{2}{\pi} \frac{N+1}{\lambda_{\max }} \sqrt{1-\left(\lambda / \lambda_{\max }\right)^{2}} d \lambda \\
& =\frac{2}{\pi} \frac{N+1}{\lambda_{\max }^{2}} \sqrt{\lambda_{\max }^{2}-\lambda^{2}} \sqrt{\frac{m \omega}{\hbar}} d x \\
& =\frac{2}{\pi \hbar} \frac{N+1}{\lambda_{\max }^{2}} p d x
\end{aligned}
$$

The right hand side is evaluated on the upper branch of the classical orbit corresponding to the maximum allowed energy. We can kick this up another notch by using Eq.(12):

$$
\text { for large } \mathrm{N}, \quad d F \approx \frac{1}{\pi \hbar} p d x .
$$

In the limit of large $N$, the cumulative distribution becomes the classical action differential form, normalized by Planck's constant. When integrated, this provides the value of action for the orbit of maximum energy.

$$
\int_{x \in\left[-x_{\max }, x_{\max }\right]} d F=N+1 \approx \frac{1}{\pi \hbar} \int_{H=E_{\max }, p \geq 0} p d x=\frac{1}{\hbar} \frac{1}{2 \pi} \oint p d x=\frac{1}{\hbar} I
$$

Combining this with Eq.(2), we obtain a reasonable, though not exact, result.

$$
E_{\text {max }} \approx(N+1) \hbar \omega
$$




\section{Synopsis}

We have truncated the matrices representing annihilation and creation operators in the number representation of the harmonic oscillator's Hilbert space. The truncated position operator possesses a discrete spectrum - points of an " $N$-space" corresponding to the roots of the Hermite polynomial $H_{N+1}$. Their range is just large enough to accomodate classical motion at high energy. In the process, a large pool of negative energy must be introduced which affects only the last eigenstate, $|N\rangle$, perhaps because it is concentrated near the endpoints of $N$-space. Calculations suggest that, in the limit of large $N$, the global distribution of points approaches a limit related to the action differential form.

\section{References}

[1] Milton Abramowitz and Irene Stegun (ed.). Handbook of Mathematical Functions. Dover Press, 1965.

[2] Albert Messiah. Quantum Mechanics. John Wiley \& Sons, Inc., New York, 1966.

[3] Leo Michelotti. Intermediate Classical Dynamics with Applications to Beam Physics. John Wiley \& Sons, Inc., New York, 1995.

[4] Gabor Szegö. Orthogonal Polynomials. American Mathematical Society, New York, 1939. 


\section{A Three matrix lemmas}

Let $\underline{a}=\left(a_{1}, a_{2}, a_{3}, \ldots\right)$ and $\underline{b}=\left(b_{1}, b_{2}, b_{3}, \ldots\right)$ be two infinite, indexed set of complex numbers none of which is zero. Define $D_{n}(\underline{a}, \underline{b} ; z), n=1,2,3, \ldots$ to be the $n^{\text {th }}$ degree polynomials in $z$ given by the determinant of the finite, $n \times n$ tridiagonal matrix,

$$
D_{n}(\underline{a}, \underline{b} ; z) \equiv\left|\begin{array}{ccccccc}
z & a_{1} & 0 & 0 & \ldots & 0 & 0 \\
b_{1} & z & a_{2} & 0 & \ldots & 0 & 0 \\
0 & b_{2} & z & a_{3} & \ldots & 0 & 0 \\
0 & 0 & b_{3} & z & \ldots & 0 & 0 \\
\vdots & \vdots & \vdots & \vdots & \ddots & \vdots & \vdots \\
0 & 0 & 0 & 0 & \ldots & z & a_{n-1} \\
0 & 0 & 0 & 0 & \ldots & b_{n-1} & z
\end{array}\right| .
$$

Note that only the first $n-1$ components of $\underline{a}$ and $\underline{b}$ are used in $D_{n}$. These polynomials obey the following three lemmas.

Lemma 1. For $n \geq 3$, the polynomials $D_{n}$ obey the recursion relation,

$$
D_{n}(\underline{a}, \underline{b} ; z)=z D_{n-1}(\underline{a}, \underline{b} ; z)-a_{n-1} b_{n-1} D_{n-2}(\underline{a}, \underline{b} ; z)
$$

Proof: This follows by applying Cramer's rule to the last row of the determinant.

Lemma 2. $D_{n}(\underline{a}, \underline{b} ; z)$ is even or odd parity accordingly as $n$ is itself even or odd. That is, $D_{n}(\underline{a}, \underline{b} ;-z)=(-1)^{n} D_{n}(\underline{a}, \underline{b} ; z)$.

Proof: The statement is easily established for small values of $n$ : for example,

$$
D_{1}(\underline{a}, \underline{b} ; z)=z, \quad D_{2}(\underline{a}, \underline{b} ; z)=z^{2}-a_{1} b_{1}, \quad \text { and } D_{3}(\underline{a}, \underline{b} ; z)=z^{3}-\left(a_{1} b_{1}+a_{2} b_{2}\right) z .
$$

To prove the lemma, we proceed by induction. Suppose it is true for $D_{1}, D_{2}, \ldots, D_{n-1}$. Then, combining this with Lemma 1 , we have

$$
\begin{aligned}
D_{n}(\underline{a}, \underline{b} ;-z) & =-z D_{n-1}(\underline{a}, \underline{b} ;-z)-a_{n-1} b_{n-1} D_{n-2}(\underline{a}, \underline{b} ;-z) \\
& =-z(-1)^{n-1} D_{n-1}(\underline{a}, \underline{b} ; z)-a_{n-1} b_{n-1}(-1)^{n-2} D_{n-2}(\underline{a}, \underline{b} ; z) \\
& =(-1)^{n}\left(z D_{n-1}(\underline{a}, \underline{b} ; z)-a_{n-1} b_{n-1} D_{n-2}(\underline{a}, \underline{b} ; z)\right) \\
& =(-1)^{n} D_{n}(\underline{a}, \underline{b} ; z)
\end{aligned}
$$

From this follows the immediate corollary that $n$ is odd, then $D_{n}(\underline{a}, \underline{b} ; 0)=0$. That zero is not a root of $D_{n}$ when $n$ is even, say $n=2 k$, is established by noting that $D_{2 k}(\underline{a}, \underline{b} ; z)$ contains the term $\left(a_{1} a_{3} a_{5} \ldots a_{2 k-1}\right)\left(b_{1} b_{3} b_{5} \ldots b_{2 k-1}\right)$, and we have assumed that none of these numbers is zero.

Lemma 3. For all $n=1,2,3, \ldots: \quad D_{n}(\underline{a},-\underline{b} ; i z)=i^{n} D_{n}(\underline{a}, \underline{b} ; z)$.

Proof: The proof is similar to that of Lemma 2. Again, the statement is established by inspection when $n=1,2$, or 3. For the inductive step, we demonstrate as before that if it is true for $D_{1}, D_{2}, \ldots, D_{n-1}$, then it must be true for $D_{n}$.

$$
\begin{aligned}
D_{n}(\underline{a},-\underline{b} ; i z) & =i z D_{n-1}(\underline{a},-\underline{b} ; i z)+a_{n-1} b_{n-1} D_{n-2}(\underline{a},-\underline{b} ; i z) \\
& =i z i^{n-1} D_{n-1}(\underline{a}, \underline{b} ; z)+a_{n-1} b_{n-1} i^{n-2} D_{n-2}(\underline{a}, \underline{b} ; z) \\
& =i^{n}\left(z D_{n-1}(\underline{a}, \underline{b} ; z)-a_{n-1} b_{n-1} D_{n-2}(\underline{a}, \underline{b} ; z)\right) \\
& =i^{n} D_{n}(\underline{a}, \underline{b} ; z)
\end{aligned}
$$

The corollary here is that if $z_{o}$ is a root of $D_{n}(\underline{a}, \underline{b} ; z)$, then $i z_{o}$ must be a root of $D_{n}(\underline{a},-\underline{b} ; z)$. 